\title{
OCORRÊNCIA DE AFLATOXINAS EM ARROZ CONSUMIDO POR MILITARES DO EXÉRCITO BRASILEIRO POR CROMATOGRAFIA EM CAMADA DELGADA E CROMATOGRAFIA LÍQUIDA DE ALTA EFICIÊNCIA
}

\author{
Incidence of aflatoxins in rice to be consumed by militaries in the brazilian army by \\ thin layer chromatography and high performance liquid chromatography methods
}

\author{
Jader Oliveira da Silva ${ }^{1}$, Lys Mary Bileski Cândido², Daiana Novello ${ }^{3}$, Cintia Machado ${ }^{4}$
}

\begin{abstract}
RESUMO
Fungos do gênero Aspergillus como A. flavus e A. parasiticus são capazes de produzir metabólitos secundários tóxicos denominados aflatoxinas. Estas espécies podem se desenvolver rapidamente em cereais sob condições favoráveis. A partir do conhecimento dos riscos à saúde decorrentes da presença de aflatoxinas em alimentos, foi realizado um levantamento para avaliar a presença de aflatoxinas em arroz beneficiado polido tipo I, destinado ao consumo dos militares do Exército Brasileiro. As amostras foram coletadas no armazém do $5^{\circ}$ Batalhão de Suprimento do Exército Brasileiro, Curitiba-PR no período de novembro de 2003 a fevereiro de 2004 e avaliada a ocorrência de aflatoxinas $B_{1}, B_{2}, G_{1}$ e $G_{2}$, utilizando-se as técnicas de Cromatografia em Camada Delgada (CCD) e Cromatografia Líquida de Alta Eficiência (CLAE). Objetivou-se, neste trabalho, contribuir com o controle de qualidade dos alimentos consumidos pelos militares da $5^{\circ}$ Região Militar do Exército Brasileiro, no que se refere ao controle dos níveis de aflatoxinas e comparar as técnicas de CCD e CLAE. Do total de 30 amostras analisadas por cromatografia em camada delgada, não foi verificada em nenhuma a presença de aflatoxinas. De 26 amostras analisadas por cromatografia líquida de alta eficiência, seis $(23,07 \%)$ apresentaram positividade para aflatoxina $\mathrm{B}_{1}(\mathrm{AFB} 1)$, com níveis entre 0,54 e $2,04 \mu \mathrm{g} / \mathrm{kg}$ e uma $(3,84 \%)$ apresentou presença de aflatoxina $\mathrm{B}_{2}\left(\mathrm{AFB}_{2}\right)$ com $1,84 \mu \mathrm{g} / \mathrm{kg}$.
\end{abstract}

Termos para indexação: Fungos, Aspergillus, arroz.

\begin{abstract}
Aspergillus are fungal species like a A flavus and A parasiticus and they are able to develop secondary metabolites called mycotoxins. They grow quickly under ideal conditions. From the moment one discovered its danger for health resulted from the presence of aflatoxins in foods, an achievement was carried out to evaluate the presence of a mycotoxins lifiting in benefited polished rice type 1, used by Brazilian army. The samples were collected at " $5^{\circ}$ Batalhão de Suprimento" (army departament) from November 2003 to febuary 2004 when the incidence of aflatoxins $B_{1}, B_{2}, G_{1}$ and $G_{2}$ was measured by thin layer chromatography (TLC) and high performance liquid chromatography (HPLC) methods. This work had the aim of comparing the thin layer chromatography and high performance liquid chromatography techniques and contributed for the quality control of the foods to be consumed by militaries of the Brazilian army, concerning the control of the aflatoxins levels. From a total of 30 samples were tested performed by tlc, no aflatoxin was detected. From 26 samples, analysed trough high performance liquid chromatography, $6(23,07 \%)$ presented positive results for aflatoxin $B_{1}$ with $0,54-2,04 \mu \mathrm{g} / \mathrm{kg}$ and, $1(3,84 \%)$ presented a positive result for aflatoxins $\mathrm{B}_{2}$ with $1,84 \mu \mathrm{g} / \mathrm{kg}$.
\end{abstract}

Index terms: Mold, Aspergillus, rice.

(Recebido em 28 de abril de 2006 e aprovado em 20 de dezembro de 2006)

\section{INTRODUÇÃO}

Alguns fungos produzem metabólitos secundários tóxicos que, às vezes, são cancerígenos quando ingeridos tanto pelo homem como pelos animais. Esses fungos são chamados toxigênicos e os metabólitos são denominados micotoxinas (SABINO et al., 1982).

Segundo Boeing (2003), os fungos para se desenvolverem e produzirem micotoxinas necessitam de condições favoráveis, e os fatores mais importantes são: a temperatura, atividade de água e teor de umidade, $\mathrm{pH}$, composição química do alimento, taxa de oxigenação, período de armazenagem, grau de contaminação fúngica, condições físicas dos grãos ou sementes, artrópodes e interação microbiana.

Micotoxinas são metabólitos secundários tóxicos produzidos por alguns fungos filamentosos, sendo os principais representantes os dos gêneros Aspergillus,

\footnotetext{
${ }^{1}$ Mestre em Ciências Veterinárias - Laboratório de Inspeção de Alimentos e Bromatologia - 5 Batalhão de Suprimentos/Exército Brasileiro - Avenida Silva Jardim, 110 - Rebouças - 80230-000 - Curitiba, PR - jader os@hotmail.com

2Doutora, Professora - Programa de Pós-Graduação em Ciências Farmacêuticas - Universidade Federal do Paraná/UFPR - Lothario Meisner 632 Jardim Botânico - 80210-170 - Curitiba, PR - lysmary@ufpr.br

${ }^{3}$ Mestre em Ciências Veterinárias, Professora - Departamento de Nutrição/DNUT - Universidade Estadual do Centro-Oete/UNICENTRO - Rua Simeão Camargo Varella de Sá, 03 - 85040-080 - Guarapuava, PR - nutridai@hotmail.com

${ }^{4}$ Mestre em Biologia Molecular - Laboratório de Inspeção de Alimentos e Bromatologia - 5ํㅡㄹatanão de Suprimentos/Exército Brasileiro - Avenida Silva Jardim, 110 - Rebouças - 80230-000 - Curitiba, PR - cíntia_machado@yahoo.com.br
} 
Penicillium e Fusarium (INSTITUTO ADOLFO LUTZ, 2005). Entre as micotoxinas existentes, as aflatoxinas, metabólitos secundários dos fungos Aspergillus flavus, Aspergillus parasiticus e Aspergillus nomius, são as que podem causar maiores danos aos seres humanos e animais, pela sua alta toxicidade e ampla ocorrência, possuindo inclusive propriedades carcinogênicas, mutagênicas e teratogênicas (SIMIONATO et al., 2003).

Há mais de 20 tipos de moléculas de aflatoxinas e seus derivados isolados, porém os principais tipos estudados continuam sendo a $B_{1}, B_{2}, G_{1}$ e $G_{2}$ (HUSSEIN \& BRASEL, 2001). Esses compostos caracterizam-se pela elevada toxicidade que apresentam (ROSA, 1995).

As aflatoxinas têm sido identificadas como fatores envolvidos na etiologia do câncer hepático no homem, conseqüente à ingestão de alimentos contaminados (MCLEAN \& DUTON, 1995).

Os cereais são facilmente colonizados por fungos patogênicos, proporcionados pelas condições favoráveis ao fungo no campo, depois da colheita e durante a estocagem (GALVANO et al., 2005). O arroz é um cereal que faz parte do hábito alimentar do brasileiro, o que se confirma pelo alto consumo, considerando suas diferentes formas.

Várias pesquisas sobre micotoxinas em arroz vêm sendo realizadas em diversos países, porém no Brasil são poucas as ocorrências relatadas com níveis e incidências baixos, que variam de 0 a $9 \mu \mathrm{g} / \mathrm{kg}$ em CCD (NUNES et al., 2003; SILVA et al., 1996; SIMIONATO et al., 2003) em CLAE variaram de 0 a $101 \mu \mathrm{g} / \mathrm{kg}$ (DILKIN et al., 2004). A disponibilidade de um método sensível e confiável é um fator-chave para a detecção de aflatoxinas em arroz (LIPIGORNGOSON et al., 2003).

Estudos anteriores realizados por Tabata et al. (1993) sobre a contaminação com aflatoxinas na maioria dos países asiáticos, incluindo o arroz, foram feitos por $\mathrm{CCD}$, método com limite de detecção relativamente alto de $1 \mu \mathrm{g} / \mathrm{kg}$, se comparados com outras técnicas como a cromatografia líquida de alta eficiência (LIPIGORNGOSON et al., 2003).

Desde a descoberta das aflatoxinas, em 1960, diversos países adotaram limites de tolerância para essas toxinas, em produtos destinados ao consumo humano. $\mathrm{O}$ Brasil, com base nos conhecimentos então disponíveis, estabeleceu, em 1977 através do Ministério da Saúde (BRASIL, 1977), o limite de $30 \mu \mathrm{g} / \mathrm{kg}$ para a soma das frações $\mathrm{B}_{1}$ e $\mathrm{G}_{1}$ em alimentos para o consumo humano. Posteriormente, a portaria ${ }^{\circ} 183$ de 1996 do Ministério da Agricultura e da Reforma Agrária (BRASIL, 1996), estabeleceu um limite de $20 \mathrm{mg} / \mathrm{kg}$ para aflatoxinas $\mathrm{B}_{1}+\mathrm{B}_{2}$
$+\mathrm{G}_{1}+\mathrm{G}_{2} \mathrm{e}$, um limite máximo de 50mg para ração animal. Mais recentemente, o Ministério da Saúde, através da resolução RDC n.274, da Agência Nacional de Vigilância Sanitária (ANVISA), de 15 de outubro de 2002, estabeleceu o limite de $20 \mathrm{mg} / \mathrm{kg}$ para aflatoxinas totais em amendoim, pasta de amendoim e milho e $0,5 \mathrm{mg} / \mathrm{L}$ e $5,0 \mathrm{mg} / \mathrm{kg}$ para aflatoxina $M_{1}$ no leite fluido e em pó, respectivamente (BRASIL, 2002).

Objetivou-se, neste trabalho, verificar a ocorrência e quantificar aflatoxina $B_{1}, B_{2}, G_{1}$ e $G_{2}$ em arroz beneficiado, consumido por militares da $5^{\circ}$ Região Militar do Exército Brasileiro, através de CCD e por CLAE.

\section{MATERIAL E MÉTODOS}

As amostras foram coletadas de diferentes locais do lote de arroz beneficiado polido tipo 1, armazenado no $5^{\circ}$ Batalhão de Suprimento do Exército Brasileiro, CuritibaPR, no período de fevereiro a abril de 2004. Foi utilizada a metodologia proposta por Fonseca (1991) que sugere a utilização de critérios de amostragem com um expoente fracionário para os lotes, em que, para produtos ensacados, emprega-se a seguinte fórmula: $\mathrm{N}=3 \sqrt{\mathrm{n}}$, onde $\mathrm{N}$ é o número mínimo de sacos a serem amostrados e n é o número de sacos que compõem o lote. As amostras analíticas foram obtidas de um total de $48000 \mathrm{~kg}$, divididos em 10 lotes de $4800 \mathrm{~kg}$, retiradas de sacos de $50 \mathrm{~kg}$.

Desse lote, foram obtidas amostras maiores do que as desejáveis para análise, sendo então misturadas, moídas, homogeneizadas e subdivididas até obtenção das amostras de tamanho desejadas. Em seguida, as amostras sofreram processo de quarteamento para tomada de amostra analítica, que somaram um número total de 30 amostras de $500 \mathrm{~g}$ e foram armazenadas $\mathrm{a}-18^{\circ} \mathrm{C}$.

A amostra analítica foi removida de uma grande amostra, que é o acúmulo de pequenas porções tomadas de diferentes locais do lote (WHITAKER et al., 2005). Posteriormente, algumas amostras foram contaminadas com soluções padrão das aflatoxinas $\mathrm{B}_{1}, \mathrm{~B}_{2}, \mathrm{G}_{1}$ e $\mathrm{G}_{2}$, para ensaios de recuperação.

As amostras foram analisadas pelas técnicas de CLAE e CCD. As análises por cromatografia líquida de alta precisão foram realizadas no Laboratório de Alimentos (LABA) do Instituto de Tecnologia do Paraná (TECPAR), no período de julho a outubro de 2004. As análises por CCD foram realizadas no Laboratório de Inspeção de Alimentos e Bromatologia do $5^{\circ}$ Batalhão de Suprimentos do Exército Brasileiro, localizado na cidade de Curitiba-PR, no período de março a junho de 2004. Esse Batalhão possui, além do laboratório de análise de alimentos, depósito e câmaras frigoríficas para armazenagem da alimentação 
destinada ao consumo de militares da $5^{\circ}$ Região Militar do Exército, que é composta pelos estados do Paraná e Santa Catarina.

Para esse trabalho foi utilizado o método por CCD, conforme proposto por Soares \& Rodriguez-Amaya (1989), que consiste na extração das toxinas com metanol/ $\mathrm{KCl}$ 4\% (9:1), remoção de interferentes com solução de sulfato de amônio $30 \%$ e partição para clorofórmio. A quantificação de micotoxinas foi realizada por comparação visual da intensidade da fluorescência das amostras com padrões sob iluminação UV a $365 \mathrm{~nm}$, sendo que o limite de quantificação para o arroz obtido foi de $3 \mathrm{mg} / \mathrm{kg}$ para $\mathrm{AFB}_{1}, \mathrm{AFB}_{2}, \mathrm{AFG}_{1}$ e $\mathrm{AFG}_{2}$. Os autores obtiveram para aflatoxina $\mathrm{B}_{1} 4 \mathrm{mg} / \mathrm{kg}$. As análises por CLAE foram realizadas seguindo-se a metodologia proposta por Lipigorngoson et al. (2003) que utilizaram método de cromatografia líquida de alta eficiência, utilizando o sistema MERCK-HITACHI "LA CHROM". Essa metodologia fundamenta-se na extração de aflatoxinas usando uma mistura de metanol e água concentrados por meio de uma coluna de imunoafinidade. As aflatoxinas $\mathrm{B}_{1}, \mathrm{~B}_{2} \mathrm{G}_{1}$ e $\mathrm{G}_{2}$ foram separadas por cromatografia líquida, com fase móvel composta por água/acetonitrila/metanol (3:1:1), acrescidos de $119 \mathrm{mg}$ de brometo de potássio e $350 \mathrm{ml}$ de ácido nítrico $65 \%$ a fluxo de $1,0 \mathrm{ml} / \mathrm{min}$, usando coluna RP 18. Após separação, as aflatoxinas foram derivatizadas em um reator pós-coluna eletroquímico marca KOBRACELL e pesquisados por detector de fluorescência, com comprimento de onda de $360 \mathrm{~nm}$ e $425 \mathrm{~nm}$ para $B_{1}$ e $B_{2}$ e $455 \mathrm{~nm}_{\text {para }} \mathrm{G}_{1} \mathrm{e}_{2}$. Nesse trabalho o limite de quantificação foi de $0,5 \mathrm{mg} / \mathrm{kg}$ para cada aflatoxina.

A umidade do arroz armazenado se mantém em equilíbrio com a umidade relativa do ar, portanto a variação da umidade relativa do ar faz com que os grãos em determinados ambientes aumentem ou diminuam a sua umidade. Foram medidas, por meio de leitura diária, a média de umidade relativa do ar e da temperatura média pelo termohigrômetro, durante o período de armazenagem do produto, que foi de fevereiro a abril de 2004. Nesse mesmo período foi feito um levantamento das condições de temperatura e umidade na cidade de Curitiba, com dados do Instituto Tecnológico SIMEPAR.

\section{RESULTADOS E DISCUSSÃO}

Apresentam-se, na Tabela 1, apresenta os resultados obtidos da umidade da amostra de arroz analisada durante o período de estocagem.

Conforme observa-se pela Tabela 1 , o teor de umidade do arroz foi de $12,32 \% \pm 0,52$. Entre os valores analisados, nenhuma das amostras apresentaram teor médio de umidade acima dos limites permitidos na legislação. Salienta-se que amostras que apresentem valores acima das previstas legalmente são rejeitadas pelo Exército por força do regulamento. Nenhuma amostra analisada apresentou limites de umidade acima dos descritos em literatura como sendo ideais para o desenvolvimento de fungos que é de $13 \%$ segundo Pereira et al. (2002).

A umidade do grão é também influenciada pelas condições climáticas, entre elas, umidade relativa do ar e temperatura do ambiente. No trabalho em questão, a umidade relativa do ar média, dentro do armazém, apresentou-se acima da média $(75,3 \%)$ para a cidade de Curitiba, no mesmo período. Com relação à temperatura, a média obtida no armazém foi de $23,1^{\circ} \mathrm{C}$, sendo que a temperatura média da cidade de Curitiba no mesmo período foi de $23,1^{\circ} \mathrm{C}$ apresentando, portanto parâmetros idênticos.

Quanto às médias obtidas de umidade relativa do ar e de temperatura ambiente do armazém, elas demonstraram níveis inferiores aos ideais tanto para o desenvolvimento dos fungos Aspergillus e Penicillium, quanto para a produção de aflatoxinas. Através de estudo de prevalência observou-se que a contaminação de grãos por fungos aflatoxigênicos por $A$. flavus é predominante sobre o $A$. parasiticus e sua produção é favorecida por temperaturas entre $23-26^{\circ} \mathrm{C}$ e umidade relativa do ar acima de $75 \%$.

Umidade relativa do ar acima de $85 \%$ e temperatura acima de $27^{\circ} \mathrm{C}$ favorece, além do crescimento, a produção de aflatoxicinas por fungos toxigênicos (Prado et. at. 1989).

A Tabela 2 apresenta os valores de recuperação obtidos para as quatro aflatoxinas.

Tabela 1 - Teor de umidade do arroz.

\begin{tabular}{lcccc}
\hline \multicolumn{1}{c}{ Amostra } & Teor de Umidade* & Umidade permitida** & $\mathrm{S}$ & $\mathrm{CV}(\%)$ \\
\hline $\begin{array}{l}\text { Arroz beneficiado } \\
\text { polido tipo I }\end{array}$ & $12,32 \%$ & $14 \%$ & 0,52 & 4,22 \\
\hline
\end{tabular}

$\mathrm{S}=$ desvio padrão; $\mathrm{CV}=$ coeficiente de variação; * valor médio das 30 determinações; **valor de umidade máxima permitida pela legislação vigente. 
Tabela 2 - Taxa de recuperação de aflatoxinas por CCD.

\begin{tabular}{cccccc}
\hline Aflatoxina & $\begin{array}{c}\text { Quantidade } \\
\text { Adicionada } \\
(\mu \mathrm{g} / \mathrm{kg})\end{array}$ & $\begin{array}{c}\text { *Quantidade } \\
\text { recuperada } \\
(\mu \mathrm{g} / \mathrm{kg})\end{array}$ & $\begin{array}{c}\text { *Recuperação } \\
(\%)\end{array}$ & $\mathrm{S}$ & $\begin{array}{c}\mathrm{CV} \\
(\%)\end{array}$ \\
\hline $\mathrm{B}_{1}$ & 3 & 2,8 & 96,0 & 13,38 & 13,89 \\
& 5 & 4,6 & 86,8 & 18,60 & 21,46 \\
& 10 & 9,9 & 99,7 & 15,75 & 15,78 \\
$\mathrm{~B}_{2}$ & 3 & 3,2 & 107,0 & 14,69 & 13,73 \\
& 5 & 4,6 & 93,0 & 17,23 & 18,53 \\
& 10 & 10,3 & 103,0 & 28,19 & 27,17 \\
$\mathrm{G}_{1}$ & 3 & 2,6 & 89,5 & 24,41 & 27,20 \\
& 5 & 3,9 & 78,0 & 18,92 & 24,25 \\
& 10 & 8,1 & 81,0 & 19,91 & 24,58 \\
$\mathrm{G}_{2}$ & 3 & 2,8 & 95,7 & 14,84 & 15,49 \\
& 5 & 3,8 & 76,8 & 20,12 & 26,19 \\
& 10 & 8 & 80,0 & 21,50 & 26,79 \\
\hline
\end{tabular}

$\mathrm{S}=$ Desvio-padrão da média das recuperações $\mathrm{CV}=$ Coeficiente de variação; *Valor médio em $\mathrm{n} 5$.

$\mathrm{Na}$ avaliação da cromatografia do arroz polido tipo 1 observou-se que do total de 30 amostras analisadas em duplicata por $\mathrm{CCD}$, não foi verificada em nenhuma a presença de aflatoxinas $\mathrm{B}_{1}, \mathrm{~B}_{2}, \mathrm{G}_{1}$ e $\mathrm{G}_{2}$. Siminonato et al. (2003), analisando 68 amostras de arroz polido, parboilizado e integral, encontraram 2 amostras (2,9\%) com contaminação por aflatoxinas $\mathrm{B}_{1}$ com níveis de $9 \mathrm{mg} / \mathrm{kg}$ e $6 \mathrm{mg} / \mathrm{kg}$. Resultados de outros trabalhos evidenciam a pouca ocorrência com baixos níveis de aflatoxinas neste cereal (NUNES et al., 2003; SILVA et al., 1996; SOARES \& RODRIGUEZ-AMAYA, 1989).

A determinação dos níveis de recuperação foi realizada com amostras contaminadas artificialmente com 3,5 e $10 \mathrm{mg} / \mathrm{kg}$ para as quatro aflatoxinas (tabela 2). Para $\mathrm{AFB}_{1}$, as taxas médias obtidas foram de 86,8 a 99,7\%; para $\mathrm{AFB}_{2}, 93$ a $107 \%$ e para $\mathrm{AFG}_{1}$ e $\mathrm{AFG}_{2}$ as taxas médias obtidas foram de respectivamente 81 a $89,5 \%$ e 80 a $95,7 \%$. Todos estes valores encontram-se dentro dos níveis aceitáveis de recuperação (70 a 120\%) desta metodologia, sendo considerados adequados para o monitoramento da contaminação de amostras (SOARES \& RODRIGUEZAMAYA, 1989).

A Tabela 3 apresenta os resultados obtidos após análise por cromatografia líquida de alta eficiência.

$\mathrm{Na}$ Figura 1 pode-se observar o cromatograma com amostra de arroz branco polido artificialmente contaminado.
Na Figura 1, nas separações cromatográficas obtidas, observam-se os picos cromatográficos apresentados para cada aflatoxina, dentro dos respectivos tempos de retenção. As variações apresentadas para as mesmas foram consideradas normais. Neste cromatograma, os picos cromatográficos estão de acordo com níveis de recuperação esperado para o método proposto e as variações de cada pico cromatográfico e suas áreas para cada aflatoxina estão dentro do esperado.

Na Figura 2 observa-se o cromatograma de uma das amostras em que se detectou a contaminação natural de aflatoxina $B_{1}$. A concentração encontrada foi de $0,547 \mathrm{mg} / \mathrm{kg}$.

Das 26 amostras analisadas, seis $(23,07 \%)$ apresentaram positividade para aflatoxina $\mathrm{B}_{1}$, com níveis variando entre 0,54 a $2,04 \mathrm{mg} / \mathrm{kg}$ e uma $(3,84 \%)$ apresentou presença de aflatoxina $\mathrm{B}_{2}$ e nenhuma atingiu o limite permitido pela legislação do Brasil.

O limite de quantificação foi de $0,5 \mathrm{mg} / \mathrm{kg}$, para cada aflatoxina. Quanto aos coeficientes de variação obtidos, as amostras contaminadas com $5 \mathrm{mg} / \mathrm{kg}$ apresentaram maior homogeneidade que as amostras contaminadas artificialmente com 3mg/kg. Segundo Gill (1987), experimentos realizados em áreas biológicas apresentam coeficientes de variação na faixa de 5 a $50 \%$.

Lipigorngoson et al. (2003), utilizando o método de coluna de imunoafinidade/cromatografia líquida, para 


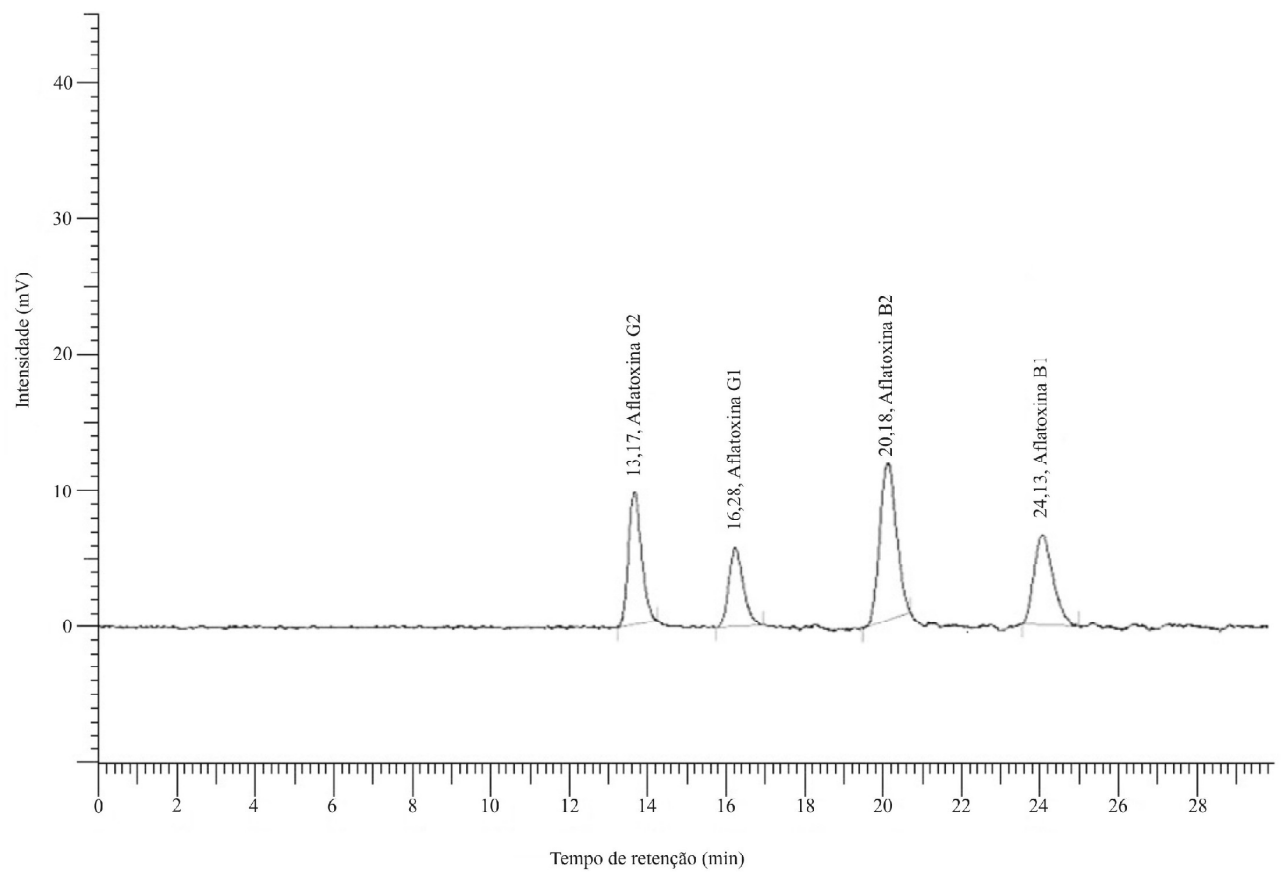

Figura 1 -Cromatograma de amostra contaminada artificialmente com 3mg/kg.

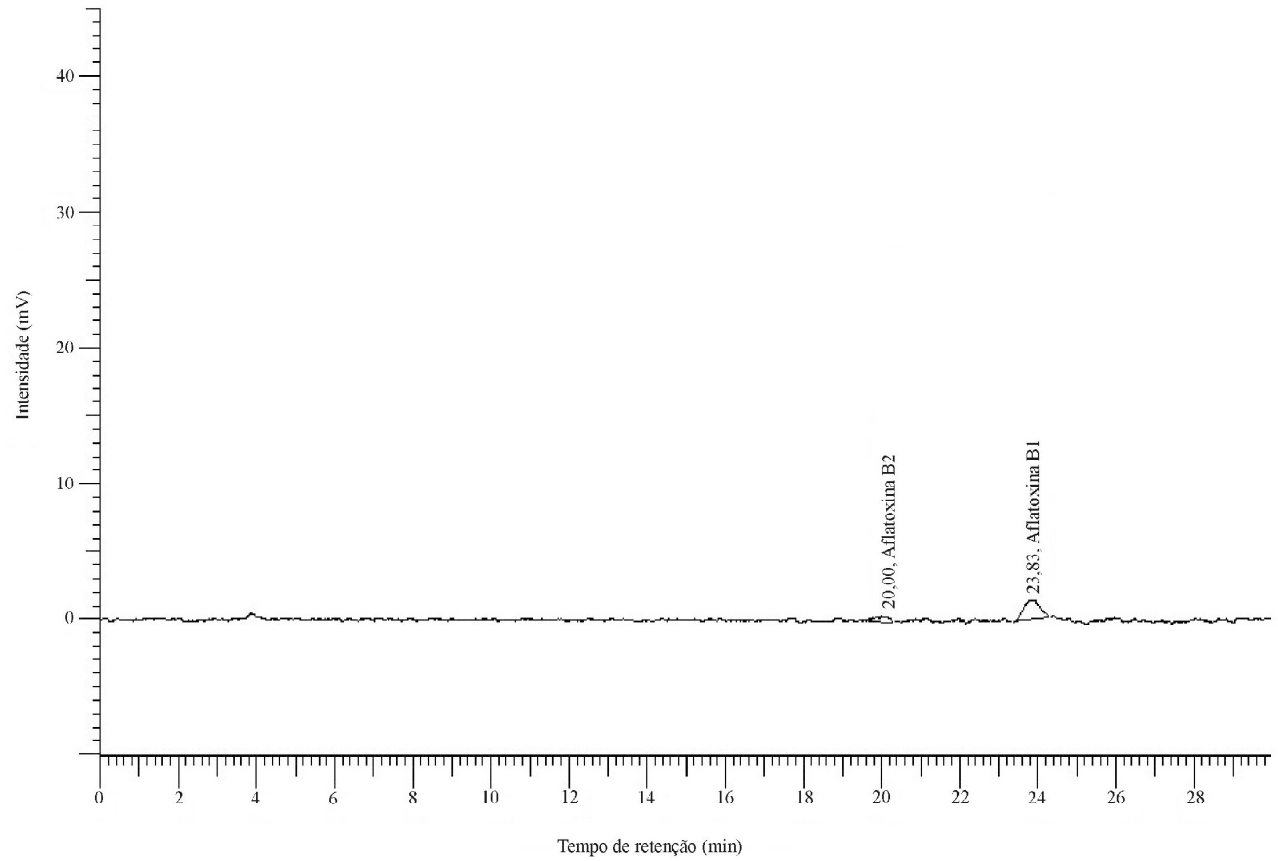

Figura 2 - Cromatograma de contaminação natural em arroz por aflatoxina $B_{1}$. 
pesquisa de aflatoxina em arroz, obtiveram limites de recuperação de $95,101,112$ e $110 \%$ para amostras contaminadas com $0,2 \mathrm{mg} / \mathrm{kg}$, e $82,79,89$ e $83 \%$ em amostras com contaminação de $0,5 \mathrm{mg} / \mathrm{kg}$ respectivamente, para $\mathrm{AFB}_{1}, \mathrm{AFB}_{2}, \mathrm{AFG}_{1}, \mathrm{AFG}_{2}$. A determinação dos níveis de recuperação foi realizada com amostras contaminadas artificialmente com 3 e $5 \mathrm{mg} / \mathrm{kg}$ de solução padrão das 4 aflatoxinas. Para $\mathrm{AFB}_{1}$ as taxas médias obtidas foram de $95,33 \%$ a $99,66 \%$; $\mathrm{AFB}_{2}-92,2$ a $112,2 \%$ e para $\mathrm{AFG}_{1}$ e $\mathrm{AFG}_{2}$ as taxas médias obtidas foram de respectivamente $91 \%$ a $99,13 \%$ e $81,63 \%$ a $86,46 \%$. A contaminação dessas amostras foi realizada para determinação da exatidão do método utilizado e foi indicada pela diferença entre o valor obtido e o valor real obtido.

Lipigorngoson et al. (2003), encontraram em 5 amostras, de um total de 20 (25\%), aflatoxina $\mathrm{B}_{1}$ com concentrações variando entre $0,1-0,3 \mathrm{mg} / \mathrm{kg}$, resultados esses, muito semelhantes aos encontrados nessa pesquisa. Observando-se para a mesma metodologia níveis de ocorrência similares, com pequena variação nos níveis de contaminação. Dilkin et al. (2004) realizando pesquisa sobre incidência de aflatoxinas em arroz, com injeção em Cromatógrafo Líquido de Alta Eficiência, encontraram positividade em $13,9 \%$, com valores inferiores aos encontrados no presente experimento, sendo que o nível médio encontrado por esses autores foi de $7,02 \mathrm{mg} / \mathrm{kg}$, mostrando-se superiores aos do presente trabalho. A diferença dos níveis de concentração, em relação a outros trabalhos, ressalta a importância das boas práticas de cultivo, transporte e armazenamento desse cereal para consumo humano.

\section{CONCLUSÕES}

O método de Soares \& Rodrigues-Amaya demonstrou ser de baixo custo, rápido e adequado para análise de rotina, com número elevado de amostras. O extrato final obtido da limpeza da amostra foi límpido e claro, apresentando um resultado sem interferentes.

Os resultados obtidos para $\mathrm{AFB}_{1}$ e $\mathrm{AFB}_{2}$, por CLAE indicam a aplicabilidade do método para a determinação de baixos níveis de contaminação em aflatoxinas, em arroz.

Os limites encontrados de aflatoxinas $\mathrm{B}_{1}$ por cromatografia líquida estiveram abaixo do limite previsto pela Legislação Brasileira porém, como já foi citado anteriormente, não se tem conhecimento se esses valores representam ou não risco significativo para o desenvolvimento do câncer hepático.

Apesar dos baixos níveis encontrados nessa pesquisa, a presença de micotoxinas em alimentos é um risco à saúde humana e animal, sendo necessárias medidas de controle e monitoramento em todos os níveis da cadeia alimentar, pelos riscos do efeito crônico da contaminação.

Baseados nesses dados, o estabelecimento de limites máximos das aflatoxinas, de acordo com os níveis internacionais, seria um passo importante para o Brasil no sentido de que, em um futuro próximo, o país não sofra prejuízos comerciais por causa de barreiras sanitárias impostas pelos países importadores.

\section{AGRADECIMENTOS}

À TECPAR pela parceria oferecida para realização desse trabalho. Ao Exército Brasileiro, por estimular o desenvolvimento técnico-científico e fornecer o apoio logístico necessário. À Pós-graduação em Ciências Veterinárias da UFPR, que permitiu a realização desse estudo, sendo parte da dissertação de Mestrado do primeiro autor.

\section{REFERÊNCIAS BIBLIOGRÁFICAS}

BOEING, C. R. Micotoxinas: causa de envenenamento alimentar. Disponível em: <http://www.crg.org.br/solucaof' 'numero18/notícia1.htmì. Acesso em: 28 jul. 2003.

BRASIL. Ministério da Agricultura e da Reforma Agrária. Portaria n. 183, art 1. Adotar regulamento técnico do MERCOSUL sobre limites máximos de aflatoxinas admissíveis no leite, amendoim e milho, aprovado pela resolução n. 56/94 do grupo Mercado comum do Sul de 1 de janeiro de 1995. Diário Oficial [da] União, Brasília, 25 mar. 1996.

BRASIL. Ministério da Saúde. Resolução n.34/76 da Comissão Nacional de Normas e padrões para alimentos. Fixa padrões de tolerância para as aflatoxinas em alimentos. Diário Oficial [da] União, Brasília, pt. I, p. 710, 19 jan. 1977. Seção I.

BRASIL. Ministério da Saúde. Resolução RDC n. 274, da ANVISA, de 15 de outubro de 2002. Diário Oficial [da] União, Brasília, 16 out. 2002.

DILKIN, M.; ARAÚJO, D. D.; FICK, F. A.; DILKIN, P.; MALLMANN, C. A. Níveis de aflatoxinas em arroz. Santa Maria: UFSM, 2004.

FONSECA, H. Sistema de amostragem para análise de aflatoxinas em grãos. Revista de Microbiologia, São Paulo, v. 21, n. 2, p. 66-70, 1991.

GALVANO, F. et al. Mycotoxins in the human food Chain. In: DIAZ, D. E. The micotoxin blue book. Nottingham: Nottingham University, 2005. p. 187-223. 
GILL, J. L.Design and analysis of experiments in the animal and medical sciences. Ames: The Lowa State University, 1987. v. 1, 411 p.

HUSSEIN, S. H.; BRASEL, J. M. Toxicity, metabolism and impact of mycotoxins on humans and animals. Toxicology, Ireland, v. 167, n. 2, p. 101-134, 2001.

INSTITUTO ADOLFO LUTZ. Métodos físico-químicos para analise de alimentos. 4. ed. Brasília, DF: Editora MS, 2005.

LIPIGORNGOSON, S.; ALI, N.; YOSHIZAWA, T. Limited survey for aflatoxin contamination of rice imported into Japan. Micotoxins, Kagawa, v. 53, n. 2, p. 95-101, 2003.

MCLEAN, M.; DUTTON, M. F. Cellula interactions and metabolism of aflatoxin: an update. Pharmacology and therapeutics, London, v. 65, n. 2, p. 163-192, 1995.

NUNES, I. L. et al. Arroz comercializado na região sul do Brasil: aspectos micotoxicológicos e microscópicos. Ciência e Tecnologia de Alimentos, Campinas, v. 23, n. 2, p. 190-194, 2003.

PEREIRA, M. M. G.; CARVALHO, E. P.; PRADO, G. Crescimento e produção de aflatoxinas por Aspergillus flavus e Aspergillus parasiticus. Boletim do CEPPA, Curitiba, v. 20, n. 1, p. 141-156, jan./jun. 2002.

PRADO, G.; MATTOS, S. V. M.; PEREIRA, E. C. Níveis de aflatoxinas em alguns alimentos consumidos em Belo horizonte no período de 1983 a 1988. Ciência e Tecnologia de Alimentos, Campinas, v. 9, n. 2, p. 138-147, 1989.

ROSA, M. F. A. P. Desenvolvimento de metodologia analítica para determinação de aflatoxina B1, aflatoxicol M1 e aflatoxicol por Cromatografia por camada delgada $\mathrm{e}$ cromatografia líquida de alta eficiência em amostras de patê de fígado industrializado. 1995. 60 f. Dissertação (Mestrado em Microbiologia Veterinária) - Universidade Federal Rural do Rio de Janeiro, Rio de Janeiro, 1995.

SABINO, M.; INOMATA, E. I.; LAMARDO, L. C. A. Variação dos níveis de aflatoxina B1 em pasta de amendoim e paçoca consumidas no estado de São Paulo. Revista do Instituto Adolfo Lutz, São Paulo, v. 42, n. 1, p. 39-44, 1982.

SILVA, S. C.; OLIVEIRA, J. N.; CALDAS, E. D. Aflatoxinas em alimentos comercializados no Distrito Federal de 1985 a 1995. Revista do Instituto Adolfo Lutz, São Paulo, v. 56, n. 2, p. 47-52, 1996.

SIMIONATO, E. M. R. S.; ASTRAY, R. M.; SYLOS, C. M. Ocorrência de ocratoxina A e aflatoxinas em arroz. Revista do Instituto Adolfo Lutz, São Paulo, v. 62, n. 2, p. 123-130, 2003.

SOARES, L. M. V.; RODRIGUEZ-AMAYA, D. B. Survey of aflatoxins, ochratoxin A, zearalenone and sterigmatocystin in some Brazilian foods by using multi-toxin thin-layer chromatographic method. Journal of Association of Official Analytical Chemists International, Washington, v. 72, n. 1, p. 22-26, 1989.

TABATA, S.; KAMIMURA, H.; IBE, A.; HASHIMOTO, H.; IIDA, M.; TAMURA, Y.; NISHIMA T. Aflatoxin contamination in foods and foodstuffs in Tokio: 1986-1990. Journal of Association of Official Analytical Chemists International, Washington, v. 76, n. 1, p. 32-35, 1993.

WHITAKER, T. B.; SLATE, A. B.; JOHANSON, A. S. Sampling feeds for mycotoxin analisis In: DIAZ, D. E. The micotoxin blue book. Nottingham: Nottingham University, 2005. p. 1-21. 